\title{
Unidade de terapia intensiva neonatal (utin): A importância na sobrevida dos recém nascidos
}

Neonatal intensive care unit (nicu): The importance in survival of newborns

Unidad de cuidados intensivos neonatales (ucin): La importancia en la supervivencia de los recién nacidos

Recebido: 07/05/2021 | Revisado: 12/05/2021 | Aceito: 18/05/2021 | Publicado: 04/06/2021

Gisele de Jesus Batista

ORCID: https://orcid.org/0000-0001-9832-3307

Universidade de Gurupi, Brasil

E-mail: gysabatysta@gmail.com

Carla Thailenna Jorge Pereira

ORCID: https://orcid.org/0000-0001-6278-7564

Universidade de Gurupi, Brasil

E-mail: thailenna1991@gmail.com

Fernanda Roques Felipe

ORCID: https://orcid.org/0000-0002-1661-6876

Universidade de Gurupi, Brasil

E-mail: nandaroquesf@gmail.com

Kassio Maluar Gonsalves Luz

ORCID: https://orcid.org/0000-0002-4574-0251

Universidade Federal do Tocantins, Brasil

E-mail: kassiomaluar@gmail.com

Milena Matos da Cruz

ORCID: https://orcid.org/0000-0002-9875-8906

Instituto Presidente Antonio Carlos, Brasil

E-mail: milenamc_5@gmail.com

Deyla Jordana de Oliveira Silva

ORCID: https://orcid.org/0000-0001-7543-2690

Instituto Presidente Antonio Carlos, Brasil

E-mail: deylajordana@ hotmail.com

Kairo Sairo Porto de Melo

ORCID: https://orcid.org/0000-0002-3731-894X

Universidade de Gurupi, Brasil

E-mail: kairosairo@gmail.com

Natália Rose de Almeida Leite Furtado

ORCID: https://orcid.org/0000-0002-6907-0037

Instituto Presidente Antonio Carlos, Brasil E-mail: nataliaralf94@gmail.com

Daniela de Souza Silva

ORCID: https://orcid.org/0000-0001-5558-5980 Universidade de Gurupi, Brasil

E-mail: danielassilva@unirg.edu.br

Laura Costa Gomes

ORCID: https://orcid.org/0000-0002-6224-5826 Instituto Presidente Antonio Carlos, Brasil E-mail: Er1802@hotmail.com

Juciely Magalhaes da Silva

ORCID: https://orcid.org/0000-0002-0845-9102

Universidade de Gurupi, Brasil

E-mail: jucielyms@unirg.edu.br

\section{Resumo}

Introdução: A taxa de mortalidade infantil teve significativa queda nas últimas décadas no Brasil e no mundo, e representa um indicador importante do desenvolvimento socioeconômico dos países. Destaca-se que contribui para aumentar a taxa de mortalidade infantil, o alto número de mortes neonatais. Nesse cenário, as Unidade de Terapia Intensiva (UTIN) configuram-se em ambiente adequado para o tratamento de recém-nascidos (RN) gravemente enfermos, possibilitando manutenção da vida e recuperação da saúde das crianças. Objetivo: Realizar uma avaliação sistematizada da literatura, evidencia a importância da Unidade de Terapia Intensa no amparo e suporte dos recém nascidos. Metodologia: a pesquisa bibliográfica procura explicar e discutir um tema com base em referências teóricas publicadas em livros, revistas, periódicos e outros. Buscam também, conhecer e analisar conteúdos científicos sobre 
determinados tema. Os dados utilizados nesta pesquisa caracterizam-se como secundários, ou seja, fundamentados em dados já tratados, retirados de livros e obras para mostrar o conceito, tratamento e suas particularidades. Resultados: A prematuridade, por exemplo, além de levar o RN a internação em UTIN ainda o expõe a diversos procedimentos dolorosos necessários para manutenção de sua vida. Sendo assim, é importante que os profissionais de saúde entendam a fisiopatologia, as manifestações clínicas do neonato, o tipo e as características da dor, visando avaliação clínica qualificada e conduta terapêutica adequada e a importância da atuação da unidade intensiva em neonato. Conclusão: A assistência à saúde da criança ainda se encontra em processo de construção, juntamente com assistência à saúde em geral, em um movimento de mudança paradigmática do modelo centrado na patologia e na criança, para um modelo de construção de redes, em prol da inclusão da família e da integralidade do cuidado.

Palavras-chave: Recém-nascido; Enfermagem; Unidade de terapia intensiva neonatal.

\begin{abstract}
Introduction: The infant mortality rate has dropped significantly in recent decades in Brazil and worldwide, and represents an important indicator of the socioeconomic development of countries. It stands out that it contributes to increase the infant mortality rate, the high number of neonatal deaths. In this scenario, the Intensive Care Units (NICU) are configured in an appropriate environment for the treatment of critically ill newborns (NB), enabling the maintenance of life and the recovery of children's health. Objective: To carry out a systematic evaluation of the literature, evidencing the importance of the Intensive Care Unit in the protection and support of newborns. Methodology: bibliographic research seeks to explain and discuss a theme based on theoretical references published in books, magazines, periodicals and others. They also seek to know and analyze scientific content on certain topics. The data used in this research are characterized as secondary, that is, based on data already treated, taken from books and works to show the concept, treatment and its particularities. Results: A prematurity, for example, in addition to taking the NB to hospitalization in the NICU still exposes him to several painful procedures necessary to maintain his life. Therefore, it is important that health professionals understand the pathophysiology, the clinical manifestations of the neonate, the type and characteristics of pain, aiming at qualified clinical evaluation and appropriate therapeutic conduct and the importance of the performance of the intensive care unit in neonates. Conclusion: Child health care is still under construction, together with health care in general, in a movement of paradigmatic change from the model centered on pathology and the child, to a model of building networks, in favor of the inclusion of the family. and comprehensive care.
\end{abstract}

Keywords: Newborn; Nursing; Neonatal intensive care unit.

\title{
Resumen
}

Introducción: La tasa de mortalidad infantil se ha reducido significativamente en las últimas décadas en Brasil y en el mundo, y representa un indicador importante del desarrollo socioeconómico de los países. Se destaca que contribuye a incrementar la tasa de mortalidad infantil, el alto número de muertes neonatales. En este escenario, las Unidades de Cuidados Intensivos (UCIN) se configuran en un ambiente adecuado para el tratamiento del recién nacido críticamente enfermo (RN), posibilitando el mantenimiento de la vida y la recuperación de la salud de los niños. Objetivo: Realizar una evaluación sistemática de la literatura, evidenciando la importancia de la Unidad de Cuidados Intensivos en la protección y apoyo al recién nacido. Metodología: la investigación bibliográfica busca explicar y discutir un tema a partir de referencias teóricas publicadas en libros, revistas, publicaciones periódicas. y otros. También buscan conocer y analizar contenidos científicos sobre determinados temas. Los datos utilizados en esta investigación se caracterizan como secundarios, es decir, basados en datos ya tratados, extraídos de libros y obras para mostrar el concepto, tratamiento y sus particularidades. Resultados: Un prematuro, por ejemplo, además de llevar al RN a la hospitalización en la UCIN todavía lo expone a varios procedimientos dolorosos necesarios para mantener su vida. Por ello, es importante que los profesionales de la salud comprendan la fisiopatología, las manifestaciones clínicas del neonato, el tipo y características del dolor, con el objetivo de una evaluación clínica calificada y una conducta terapéutica adecuada y la importancia del desempeño de la unidad de cuidados intensivos en neonatos. Conclusión: La atención de la salud se encuentra aún en construcción, junto con la atención de la salud en general, en un movimiento de cambio paradigmático del modelo centrado en la patología y el niño, a un modelo de construcción de redes, a favor de la inclusión de la familia y la atención integral.

Palabras clave: Recién nacido; Enfermería; Unidad de cuidado intensivo neonatal.

\section{Introdução}

Nos últimos anos, a assistência à saúde da criança tem sofrido grandes mudanças. Os avanços científicos, a incorporação de novas tecnologias, a participação da família no cuidado da criança, o reconhecimento da importância do trabalho multiprofissional, a preocupação com a qualidade de vida e com os direitos humanos, têm contribuído significativamente para qualificar a assistência infantil (Furtado et al., 2010). 
É importante destacar que políticas públicas de saúde, direcionadas ao tratamento precoce das pneumonias e diarreias, associadas a grande esforço na prevenção das doenças infecciosas por meio das vacinações, contribuem expressivamente para a redução da mortalidade infantil (UNICEF, 2013; Moreira; Goldani, 2010).

Nessa perspectiva, destacamos que a taxa de mortalidade de crianças teve significativa queda nas últimas décadas no Brasil. Esse fenômeno é atribuído às estratégias implementadas pelo governo federal, como ações para diminuição da pobreza, ampliação da cobertura da Estratégia Saúde da Família, ampliação das taxas de aleitamento materno exclusivo, imunização, oferta de cuidado especializado, entre outras (Ministério da Saúde, 2012).

A taxa de mortalidade de menores de cinco anos é um indicador chave do bem-estar infantil, incluindo a saúde e o estado nutricional. Além disso, representa indicador da cobertura das intervenções para a sobrevivência infantil e, mais amplamente, de desenvolvimento social e econômico. Nota-se que o mundo fez progressos substanciais, reduzindo o número de mortes infantil de 90 óbitos a cada 1.000 nascidos vivos em 1990, para 46 em 2013. Desde 1990, quase 100 milhões de crianças com idade de cinco ou menos a população atual das Filipinas foram salvas. O mundo também está a redução da mortalidade de menores de cinco mais rápido do que em qualquer outro momento durante as duas últimas décadas (United Nations Children's Fund, 2014).

Apesar desse resultado, nota-se que o Brasil e diversos países ainda apresentam fragilidades em relação a proteção e promoção da saúde da criança. A sobrevivência da criança ainda continua uma preocupação urgente. O número de mortes infantis é alarmante. Para se ter ideia, entre 1990 e 2013, 223 milhões de crianças em todo o mundo morreram antes de seu quinto aniversário (UNICEF, 2014). Para acabar com as mortes infantis evitáveis, são necessárias melhorias na prevenção de doenças e tratamento, nutrição adequada, água e saneamento básico e educação de qualidade. Se as tendências atuais persistirem, entre 2015 e 2028 cerca de 35 milhões de crianças morrerão por causa evitáveis (UNICEF, 2013).

Sendo assim, torna-se imprescindível implementar intervenções para combater a inversa e significante associação entre nível socioeconômico e mortalidade infantil, que no Brasil se expressa tanto entre regiões, como no âmbito dos estados e municípios (Santos et al., 2014).

\section{Metodologia}

Este Estudo trata-se de uma revisão bibliográfica acerca da importância de Unidade de Tratamento Intensivo na sobrevida dos neonatos. Segundo Martins (2001) a pesquisa bibliográfica procura explicar e discutir um tema com base em referências teóricas publicadas em livros, revistas, periódicos e outros. Buscam também, conhecer e analisar conteúdos científicos sobre determinados tema.

Os dados utilizados nesta pesquisa caracterizam-se como secundários, ou seja, fundamentados em dados já tratados, retirados de livros e obras para mostrar o conceito, condução e suas particularidades.

Portanto a pesquisa será caracterizada em acervos bibliográficos e revisões bibliográficas com base em artigos científicos e periódicos documentais que segundo Fachin(2005) e Marconi e Lakatos (2015) são todas as obras escritas e matérias constituídas por dados primários ou secundários que pelos quais possibilitem fonte de informação ao autor e leitores dos mesmos.

Foram pesquisados artigos em website da Biblioteca Virtual em Saúde (BVS), nas bases de dados da Medical Literature Analysis and Retrieval System Online (MEDLINE®),Scientific Electronic Library Online (SciELO), e das fontes oficiais: Ministério da Saúde, e World Health Organization(WHO). Foram acessados palavras chaves em Ciências da Saúde (DeCS) os termos: Recém-Nascido. Enfermagem. Unidade de Terapia Intensiva Neonatal. 
Para a realização desse projeto será utilizado o editor de texto WORD para elaboração e sistematiçao das informações pesquisadas em plataformas virtuais. Analisamos neste projeto dados conceituais e de explanação das interfaces e sistematização da intrigante atividade das UTIN, utilizando periódicos pesquisas em bases de dados.

As limitações dessa pesquisa estão basicamente na paralisação de novas publicações sobre $U T I N$ 'S no ano vigente. A COVID-19 desacelerou as pesquisas de modo geral, pois todos os olhos foram virados para a patologia que assola nossa atualidade

\section{Resultados e Discussão}

Nessa perspectiva, destacamos que contribui para aumentar a taxa de mortalidade infantil, o alto número de mortes neonatais (IBGE, 2010). O período neonatal tem início logo após o nascimento e termina após 28 dias completos de vida da criança. As mortes neonatais (mortes entre nascidos vivos durante os primeiros 28 dias completos de vida) podem ser subdivididas em mortes neonatais precoces, que ocorrem durante os primeiros sete dias de vida, e mortes neonatais tardias, que ocorrem após o sétimo dia, mas antes de 28 dias completos de vida (MS, 2009).

Assim, consolida-se como grande desafio para qualificar a assistência em saúde, a redução da mortalidade neonatal. Para se alcançar esse objetivo, são necessárias medidas abrangentes que incluam melhorias na assistência pré-natal, no parto e ao recém-nascido (RN) (UNICEF, 2013; Lourenço, Brunken; Luppi, 2013).

Nesse cenário, destacamos que a Unidade de Terapia Intensiva Neonatal (UTIN), pelo seu grande aparato tecnológico e equipes multiprofissionais com alta qualificação técnica e também científica, configura-se ambiente adequado para o tratamento de RN gravemente enfermo, visando, entre outras questões, a manutenção da vida e recuperação da saúde dessas crianças (Perlin; Oliveira; Gomes, 2011).

Dessa maneira, a UTIN caracteriza-se como uma área de assistência a recém-nascidos graves, altamente vulneráveis, que necessitam de cuidados especiais e contínuos, o que exige dos profissionais de saúde grande conhecimento científico, habilidade técnica e capacidade de realizar avaliações particularmente criteriosas desses pacientes (Duarte; Sena; Xavier, 2009; Silva; Silva; Christoffel, 2009; Duarte; Ellensohn, 2007). Além disso, é indispensável aos profissionais de saúde habilidades para lidar com a família do RN hospitalizado, pois ela comumente fica exposta a momentos de frustração e sofrimento (Santos et al., 2012).

São classificados como RN de risco, aqueles que se apresentam clinicamente graves, ou seja, que estão em risco de morte (Brasil, 2012b). Nessa perspectiva, é importante compreender que diversas situações podem levar o RN depender de cuidados intensivos, dentre elas destaca-se a prematuridade, infecções, problemas respiratórios e circulatórios, intercorrências no parto e/ou durante a gestação, entre outros (Santos et al., 2014; Brasil, 2012).

Os altos percentuais de mortes por causas evitáveis, entre recém-nascido, sugerem problemas de acesso aos serviços de saúde, cobertura e/ou na qualidade da assistência prestada. Sendo assim, torna-se indispensável o controle da gravidez, destacando-se possíveis falhas na qualidade do pré-natal (Silva et al., 2013).

Frente aos dados anteriormente apresentados, acreditamos que conhecer o perfil das internações de RN em UTIN pode favorecer o desenvolvimento de estratégias direcionadas a promoção e proteção da saúde da criança, e favorecer a implementação de ações no pré-natal que estejam direcionadas à prevenção de agravos à saúde da criança. Consequentemente, pode reduzir o número de internações em UTIN e corroborar a eliminar o sofrimento da família que experiência a situação de ter e conviver com RN hospitalizado.

A evolução histórica da participação da criança na sociedade, assim como os cuidados à saúde voltados a essa população, apresentou avanços, tendo como reflexo a redução da mortalidade infantil e a implantação de diversas políticas 
públicas de saúde. Todavia, muitas ações ainda precisam ser pontuadas, discutidas em profundidade, reorganizadas e pactuadas para que estes avanços possam realmente refletir as reais necessidades da população infantil (Araújo, et al., 2014).

Nesse cenário, diversas situações podem levar a criança a depender de cuidados de saúde especializados. A prematuridade, por exemplo, além de levar o RN a internação em UTIN ainda o expõe a diversos procedimentos dolorosos necessários para manutenção de sua vida. Sendo assim, é importante que os profissionais de saúde entendam a fisiopatologia, as manifestações clínicas do neonato, o tipo e as características da dor, visando avaliação clínica qualificada e conduta terapêutica adequada (Santos; Pereira; Santos; Santana, 2012).

É válido destacar que a hospitalização de RN em UTIN implica em consequências à criança e à sua família. A hospitalização é um evento estressante que altera a dinâmica familiar (Santos et al, 2015; Nóbrega et al, 2010). Nas últimas décadas, a enfermagem tem ampliado o número de pesquisas que abordam o tema família frente a suas experiências com o surgimento de doença, hospitalização entre outros. Nessa perspectiva, destacamos a importância de estudos capazes de despertar reflexão e mudanças na prática assistencial dos enfermeiros, contribuindo para ajudar a fortalecer as famílias em suas experiências de sofrimento na situação de doença infantil (Wright, Leahey, 2010).

Para família, a hospitalização do RN em UTIN configura-se em sofrimento, por isso os profissionais de saúde, que atuam nessas unidades, devem alicerçar suas práticas no acolhimento, interações, trabalho em equipe, resolutividade, estabelecimento de vínculos e responsabilizações (Santos et al., 2012; Santos; Silva; Santana; Santos, 2012b).

Durante a hospitalização da criança, os pais sentem medo de perder o vínculo afetivo com o filho devido à interrupção do dia-a-dia e à distância, contudo, permanecer junto da criança, ameniza o sofrimento gerado por sua hospitalização (Carvalho et al, 2009; Molina et al, 2009).

A relação entre família e profissional da saúde deve acontecer de modo que contribua para uma maior compreensão, por parte da família, do mundo da UTIN, já que isso favorece o sucesso do tratamento e a superação da crise vivenciada pela família, durante o período de hospitalização da criança (Molina et al, 2009).

É importante que os profissionais forneçam informações e orientações aos familiares sobre as necessidades e o tratamento da criança. Compreender esse processo favorece a construção de vínculo entre a família e equipe de saúde. Apoiar, respeitar e acolher a família, no ambiente hospitalar, contribui para reduzir o sofrimento dessas pessoas que precisam conviver com a doença e hospitalização da criança (Santos et al., 2015; Araújo et al, 2010; Sousa, Gomes, Santos, 2009).

\section{Considerações Finais}

Nota-se que o preparo da mãe para o cuidado do filho, na Unidade Neonatal, é favorecido pela atuação de profissionais de enfermagem que consideram às necessidades dessas mulheres, sem impor seu conhecimento de maneira verticalizada. As mães reconhecem como importantes as informações compartilhadas pela equipe de enfermagem e acreditam que isso as capacitam para cuidar do bebê, no domicílio, trazendo-lhes segurança no desempenho do papel de cuidadora e favorecendo sua adaptação às necessidades do RN (Couto; Praça, 2012).

Por outro lado, conduta fria, impessoal e puramente técnica, no atendimento, contraria qualquer pressuposto de uma assistência terapêutica e humanizada. Quando o profissional de saúde não interage/estabelece comunicação com a pessoa a qual está atendendo, encarando-a como objeto, a ação benéfica dessa relação torna-se extremamente limitada (Maldonado; Canella, 2003).

Atualmente, políticas públicas têm buscado ampliar a abordagem para além do modelo biomédico, centrado na doença e seu tratamento, considerando a criança integralmente e em suas múltiplas relações, a família é valorizada como também o contexto em que a criança vive, e destacam-se princípios como o acesso aos serviços de saúde, integralidade do cuidado nos diversos níveis de atenção e a humanização da assistência (Furtado et al., 2010; Brasil, 2012). 
Nota-se, assim, que a assistência à saúde da criança ainda se encontra em processo de construção, juntamente com assistência à saúde em geral, em um movimento de mudança paradigmática do modelo centrado na patologia e na criança, para um modelo de construção de redes, em prol da inclusão da família e da integralidade do cuidado (Araújo, et al., 2014; Santos et al., 2013). Assim, ainda existem lacunas e limites no cuidado à criança, nas relações organizacionais e administrativas, no fortalecimento das políticas públicas, no modelo de processo de trabalho e no processo continuado de educação em saúde (Araújo, et al., 2014; UNICEF, 2013).

Portanto, é de grande relevância realizarmos pesquisas no campo da assistência neonatal, para que possamos juntos,levantarmos novas discussões, para que o conhecimento da importância da unidade de atendimento intensivo ao neonato seja reconhecido e aprimorado. O amparo técnico e profissional nos atendimentos aos pacientes neonatos, em situação de saúde vulnerável, é imprescindível para que consigamos diminuir a taxa de mortalidade nesses recém nascidos.

\section{Referências}

Almeida, A. C. et al. (2012). Fatores de risco maternos para prematuridade em uma maternidade pública de Imperatriz-MA. Rev Gaúcha Enferm. 33(2), 86-94

Anversa, Et al. (2012). Qualidade do processo da assistência pré-natal: unidades básicas de saúde e unidades de Estratégia Saúde da Família em município no Sul do Brasil. Cad. Saúde Pública, 28(4):789-800.

Araújo J. P., et al.(2014). História da saúde da criança: conquistas, políticas e perspectivas. Rev Bras Enferm. 67(6):1000-7.

Basso, C. G., Neves, E. T. \& Silveira, A. (2012). Associação Entre Realização De Pré-Natal E Morbidade Neonatal. Texto Contexto Enferm, 21(2), 269-76.

Benites, P. T., \& Nunes, C. B.(2006). Conhecendo o perfil do recém-nascido em uma unidade de terapia intensiva. Ensaios e Ciência: Ciências Biológicas, Agrárias e da Saúde, 10(3), 33- 40

Brasil.(2012). Ministério da Saúde. Atenção a saúde do recém-nascido. (2a ed.).

Brasil. (2015). Ministério da Saúde. Atenção ao pré-natal de baixo risco. Brasília: Ministério da Saúde, 2012. (Cadernos de Atenção Básica, n.18). http://bvsms.saude.gov.br/bvs/publicacoes/abcad18.pdf>.

Brasil. (2012) Ministério da Saúde. Manual aidipi neonatal. (3aed.), 6, 111-113

Brasil.(2009). Ministério da Saúde. Manual de Vigilância do Óbito Infantil e Fetal e do Comitê de Prevenção do Óbito Infantil e Fetal. (2a ed.).

Carmo, et al. (2004). Procedimentos de enfermagem em UTI neonatal. Editora FIOCRUZ,.

Carvalho, J. B. L. et al.(2009). Representação social de pais sobre o filho prematuro na Unidade de Terapia Intensiva Neonatal. Rev Bras Enferm. 62(5):734-8. http://www.scielo.br/pdf/reben/v62n5/14.pdf.

Cassiano et al. (2014). Saúde materno infantil no Brasil: evolução e programas desenvolvidos pelo Ministério da Saúde. Revista do Serviço Público Brasília 65 (2): $227-244$.

Costa et al.(2014). A Maternidade e a Paternidade: O Olhar do Casal Adolescente. Rev Bras Promoç Saúde, 27(1): 101-108.

Duarte, A. P. P., \& Ellensohn. L. (2007). A operacionalização do processo de enfermagem em terapia intensiva neonatal. $R$ Enferm UERJ, 15(4):521-6.

Duarte, E. D., Sena, R. R., \& Xavier, C. C. (2009). Processo de trabalho na Unidade de Terapia Intensiva Neonatal: construção de uma atenção orientada pela integralidade. Rev. esc. enferm. USP. 43(3), 647-654.

Duarte, S. J. H., \& Almeida, E. P.(2014). O papel do enfermeiro do programa saúde da família no atendimento pré- natal. R. Enferm. Cent. O. Min. 4(1):10291035 .

Dumas, A., Lejuene, C., \& Simmat-Durand, L. (2014).Tobacco, alcohol, marijuana and pregnancy: Whith women are at risk? Medline, 26(5), 603-12.

Furtado, M. C. C. el al. (2010). Avaliação da atenção ao recém-nascido na articulação entre maternidade e rede básica de saúde. Rev. Eletr. Enf. 12(4):640-6. http://dx.doi.org/10.5216/ree.v12i4.7625.

Harper et al. (2014). Defining an abnormal first stage of labor based on maternal and neonatal outcomes. 536.e3 American Journal of Obstetrics \& Gynecology.

IBGE. (2010). Intituto Brasileiro de Geografia e Estatística. Observações sobre a evolução da mortalidade no Brasil: o passado, o presente e perspectivas.

Kassada et al.(2013). Prevalência do uso de drogas de abuso por gestantes. Acta Paul Enferm. 2013, 26(5):467-71.

Lourenço, E. C., Brunken, G. S., \& Luppi, C. G.(2013). Mortalidade infantil neonatal: estudo das causas evitáveis em Cuiabá, Mato Grosso, 2007*. Epidemiol. Serv. Saúde, 22(4):697-706. 
Maldonado, M. T., \& Canella, P. (2003). Resources relationship to health professionals: a good communication with clients and their families in medical offices, clinics and hospitals. Reichamann \& Affonso.

Molina, R. C. M., Fonseca, E. L., Waidman, M. A. P., \& Marconi, S. S. (2009). LA percepção da família sobre sua presença em Unidade de Terapia Intensiva Pediátrica e Neonatal. Rev Esc Enferm USP. 43(3):630-8.

Moreira, M., \& Goldani, M. Z. (2010). A criança é o pai do homem: novos desafios para a área de saúde da criança. Ciência \& Saúde Coletiva, 15(2):321-327.

Nelson et al. (2014). Early Pregnancy Changes in Bacterial Vaginosis-Associated Bacteria and Preterm Delivery. 28(2), 88-96.

Nóbrega, R. D., Collet, N., Gomes, I. P., Holanda, E. R., \& Araújo, Y. B. (2010). Criança em idade escolar hospitalizada: significado da condição crônica. Texto Contexto Enferm. 19(3):425-33.

Perlin, D. A., Oliveira, S. M., \& Gomes, G. C. (2011). A criança na unidade de terapia intensiva neonatal: impacto da primeira visita da mãe. Rev. Gaúcha Enferm. 32(3), 458-464.

Pimentel, A. (2001). O método da análise documental: seu uso numa pesquisa historiográfica. Cadernos de Pesquisa, 114.

Pizzo et al. (2014). Mortalidade infantil na percepção de gestores e profissionais de saúde: determinantes do seu declínio e desafios atuais em município do sul do Brasil1. Saúde Soc. 23(3), 908-918.

Ramos, H. A. C., \& Cuman, R. K. N. (2009). Fatores de risco para prematuridade: Pesquisa Documental. Esc Anna Nery Rev Enferm. 13 (2): 297-304

Ramos, H. A. C., \& Cuman, R. K. N. (2009). Fatores de risco para prematuridade: Pesquisa Documental. Esc Anna Nery Rev Enferm. 13 (2): 297-304.

Salge et al.(2009). Fatores maternos e neonatais associados à prematuridade. Rev. Eletr. Enf.. 11(3):642-6.

Santos et al. (2014). Óbitos infantis evitáveis nas coortes de nascimentos de Pelotas, Rio Grande do Sul, Brasil, de 1993 e 2004 . Cad. Saúde Pública, 30(11):2331-2343.

Santos et al. (2013.) Reflexos da hospitalização da criança na vida do familiar acompanhante. Rev Bras Enferm, 66(4): 473-8.

Santos et al. (2015). When the communication is harmful in the encounter between health professional and family of hospitalized child. Enfermaria Global, 216-226,

Santos, et al. (2012). Grupo de suporte como estratégia para assistência de enfermagem à família de recém-nascidos hospitalizados. Rev. Eletr. Enf. 14(1):42-

Santos, L. M., Pereira, M. P., Santos, L.F.N., \& Santana, R. C. B. (2012).Avaliação da dor no recém-nascido prematuro em Unidade de Terapia Intensiva. Rev Bras Enferm, 65(1): 27-33.

Santos, L. M., Silva, C. L. S., Santana, R. C. B., \& Santos, V. E. P. (2012). Vivencias paternas durante a hospitalizacao do recem-nascido prematuro na Unidade de Terapia Intensiva Neonatal. 65(5): 788-94.

Silva, et al. (2013). Validade, confiabilidade e evitabilidade da causa básica dos óbitos neonatais ocorridos em unidade de cuidados intensivos da Rede NorteNordeste de Saúde Perinatal. Cad. Saúde Pública, 29(3):547-556.

Silva, L. J., Silva, L. R., \& Christoffel, M. M. (2009). Tecnologia e humanização na Unidade de Terapia Intensiva Neonatal: reflexões no contexto do processo saúde-doença. Rev. esc. enferm. USP. 43(3), 684-689.

Silveira et al.(2013). Prevalência de nascimentos pré-termo por peso ao nascer: revisão sistemática. Rev. Saúde Pública. 47(5).

Simón, Y \& Aznar, T. (2015). Influye la família de origen em el embarazo no planificado de adolescentes y jóvenes?. IBECS Prog. Obstet. Ginecol.. 58(3): $118-124$.

Sousa, L. D., Gomes, G. C., \& Santos, C. P. (2009). Percepções da equipe de enfermagem acerca da importância da presença do familiar/acompanhante no hospital. Rev Enferm UERJ. 17(3):394-9.

Tocantins. (2012). Secretaria do Estado do Tocantins. Protocolo de atenção integral à saúde da mulher tocantinese. Cap. 2.

Tong et al. (2015). Accuracy of self-reported smoking cessation during pregnancy. 94(1), 106-111.

Tourinho, A. B., \& Reis, L. B. S. M. (2013). Peso ao nascer: uma abordagem nutricional. Com. Ciências Saúde. 22(4):19-30.

UNICEF (2013).United Nations Children's Fund. Committing to Child Survival: A Promise Renewed Progress Report 2013.

UNICEF (2014). United Nations Children's Fund. Levels \& Trends in Child Mortality Report 2014.

Wright, L. M., \& Leahey, M. (2010). Enfermeiras e famílias: um guia para avaliação e intervenção na família. RocA.

Yamaguchi, E. T., Cardoso, M. M. S. C., Torres, M. L. A., \& Andrade, A. G. (2008). Drogas de abuso e gravidez. Rev. Psiq. Clín. 35(1), 44-47.

Salvador, P. T. C. O. et al.2010. A formação acadêmica de enfermagem e os incidentes com múltiplas vítimas: revisão integrativa. Artigo de revisão. Revista da Escola de Enfermagem.

Smeltzer, S.C., \& Bare, B. G. (2005). Tratado de enfermagem médico-cirúrgico. (10a ed.), Guanabara-Koogan, 2005.

Teixeira, J. E. V. (2011). Acidentes com múltiplas vítimas. In: Oliveira BFM, Parolin MKF. USP. 
Research, Society and Development, v. 10, n. 6, e40910615884, 2021

(CC BY 4.0) | ISSN 2525-3409 | DOI: http://dx.doi.org/10.33448/rsd-v10i6.15884

Teixeira, J. E. V. (2011). Trauma: atendimento pré-hospitalar. Atheneu, pp 497-506.

Valentim, A., Paes, G. O., \& Carvalho, S. M. (2014). Utilizando serviços de emergência do sistema único de saúde mediante simple triage and rapid treatment. Rev. Enf. Profissional. 1(1):194-204 